\title{
Des Balkans
}

About the Balkans

Predrag Matvejević

\section{(2) OpenEdition}

Journals

Édition électronique

URL : https://journals.openedition.org/ceb/1476

DOI : $10.4000 /$ ceb. 1476

ISSN : 2261-4184

Éditeur

INALCO

Édition imprimée

Date de publication : 1 décembre 2008

Pagination : 1-11

ISBN : 978-2-85831-173-6

ISSN : 0290-7402

Référence électronique

Predrag Matvejević, « Des Balkans », Cahiers balkaniques [En ligne], 36-37 | 2008, mis en ligne le 15 mai 2012, consulté le 06 juillet 2021. URL : http://journals.openedition.org/ceb/1476 ; DOI : https://doi.org/ $10.4000 /$ ceb.1476

Ce document a été généré automatiquement le 6 juillet 2021.

\section{cc) (7) (8)}

Cahiers balkaniques est mis à disposition selon les termes de la Licence Creative Commons Attribution - Pas d'Utilisation Commerciale 4.0 International. 


\title{
Des Balkans
}

\author{
About the Balkans
}

Predrag Matvejević

1 Celui qui aborde les Balkans ne tarde pas à se rendre compte de leurs contradictions aussi bien géographiques que politiques. Est-ce une véritable péninsule ou un large bloc du continent immergé dans le bassin méditerranéen ? L'un et l'autre à la fois ou bien, selon l'endroit, soit l'un soit l'autre? Maintes mers baignent ces côtes - l'Adriatique, l'Ionienne, l'Égée avec, à ses confins, celle que l'on appelle la Noire et l'autre, plus petite, dite de Marmara. Tout le littoral n'est pas maritime. L'arrière-pays est en majeure partie montagneux. L'intérieur en est coupé de la Méditerranée. Aucune des cinq mers qui l'entourent n'avait donné de nom à ces espaces, le relief de l'intérieur l'a fait pour elles : hauteurs que les anciens géographes appelaient Haemus et catena mundi, «Le vieux Mont» (Stara planina) des Slaves, que les Turcs traduisirent en leur langue par «Balkans ».

2 Dans le passé, ils avaient également pour nom péninsule Illyrienne, Grecque, Byzantine et, il y a un peu plus d'un siècle, "Turquie d'Europe » : ceci révèle, entre autres, les diverses attributions ou appartenances de ces territoires. À la différence de ses parentes apennine et ibérique, séparées du continent par des chaînes de montagnes, Alpes et Pyrénées, la presqu'île balkanique n'offre pas, face à l'Europe centrale, de barrière difficile à franchir. Pour certains géographes et historiens, ce sont les cours d'eau - Danube, Save et Kupa - qui marqueraient des frontières au nord et à l'ouest. Quant au littoral, ce seraient, d'un côté, les golfes de Kvarner (Quarnaro), de Rijeka (Fiume) ou même de Trieste (cela vaut surtout pour les mappemondes plus anciennes). De l'autre côté, à l'est, la ligne que nous hésiterions à tracer traverserait probablement la Dobrudja et s'arrêterait non loin de l'énigmatique delta danubien. Ces délimitations sont relatives et souvent arbitraires. Ceux qui les proposent ou les ratifient sont rarement en accord les uns avec les autres. Les tracés qu'ils inscrivent sur les cartes varient d'une époque à l'autre selon les intérêts des grandes puissances étrangères ou les petites querelles intérieures.

3 Les Balkans s'identifient souvent à l'Orient de l'Europe, en fonction de l'angle sous lequel on les observe et du point de vue que l'on adopte. On a répété à maintes reprises 
que, considérée du centre de notre continent, cette « zone de turbulences » commence probablement déjà près de Munich ou des vieilles murailles de Vienne, devant lesquelles furent arrêtées les troupes ottomanes. (On cite volontiers la boutade de Metternich concernant une Vienne non seulement mitteleuropéenne, mais aussi balkanique.) Les habitants de ces deux villes, offensés, repoussent cette «frontière incertaine » vers Ljubljana et Zagreb (l'écrivain croate Miroslav Krleža a fixé, non sans malice, son point initial au centre de cette ville). Les Slovènes ou les Croates, indignés, eux aussi, la rejettent à leur tour bien plus à l'est, vers Belgrade ou Sarajevo, Skopje ou Tirana. Du côté oriental de la péninsule, d'aucuns, plus avisés, répliquent non sans quelque amertume que c'est dans les Balkans que l'Europe a pris sa naissance et que là se trouve son berceau.

4 Cet espace, plus difficile à définir qu'à délimiter, a été dans son passé sujet aux grands mouvements telluriques. Les tremblements de terre y ont été fréquents depuis les temps immémoriaux, et leurs effets dévastateurs. Il y a plus de mille ans, Joseph l'Hymnographe écrivit un émouvant Canon sur la crainte du séisme: "Du séisme, du glaive, de la rude captivité, du glissement du terrain, de la famine..., ô Maitre miséricordieux, préserve Ta ville ». Bien des villes de nos côtes furent englouties par les vagues, surgies tant de la mer que de l'histoire. Nicéphore Grégoras, témoin du crépuscule de la Byzance, en fournit une image apocalyptique: "À cette époque éclatèrent des séismes et des bouillonnements marins extraordinaires... Des maisons s'écroulèrent, ainsi que la plus grande partie des remparts de Byzance... Ils submergèrent nombre de terroirs, avec les hommes euxmêmes, les troupeaux et les attelages... Des flots débordèrent sur la terre ferme et emportèrent aussi des navires qui se trouvaient à proximité des ports ».

Certaines îles voisines disparaissaient ou changeaient de place au cours des époques mythologiques. En maints endroits, on croit encore apercevoir au fond des eaux, à proximité des rivages, les ruines d'anciens palais, des ports et des môles à côté desquels gisent des épaves, remplies de trésors fabuleux. (Inutile de chercher leurs cargaisons, elles ont déjà été emportées par les pirates appartenant à Dieu sait quelle ethnie ou tribu, peuple ou nation.) Les secousses sismiques et les variations tectoniques qu'elles provoquent ne seraient donc pas en l'occurrence de simples métaphores. Quoi qu'il en soit, d'aucuns lient ces phénomènes aux mentalités et aux humeurs des habitants d'alentour. Bien des arguments pourraient nous induire à favoriser ce genre d'hypothèses, plus séduisantes que probables. Tout en hésitant à approuver certaines conclusions qu'elles inspirent, il est recommandable d'en tenir compte, ne serait-ce qu'en littérature.

6 La question de la pluralité et de la variété démographiques est aussi ancienne que les Balkans mêmes. Elle suscite l'intérêt ou la passion, tant d'illustres savants que de simples charlatans. Nous avons pu lire de curieuses recherches faites par le chanoine de Šibenik (Sebenico) qui portait le nom latin de Georgius Sisgoreus, et un autre, croate, Juraj Šižgorić (il vivait à l'époque de la Renaissance, chantant en même temps la gloire de Venise et recueillant des contes populaires slaves). Cet érudit ecclésiastique a tenté de recenser les peuples ou les tribus balkaniques, utilisant les témoignages que nous ont laissés les historiens et géographes de l'antiquité, afin de présenter les origines, étranges ou souvent exotiques, de nos prédécesseurs: "Enchéléiens (Encheleae) Himaniens, Peucéniens (Peuciai), selon Calimaque; Sérètes, Sirapiles, Iasiens (Jasi), Andisetes (ou Sandisetes), Calophiens (Calophani) et Breuciens (Breuci) selon Pline; Noriques (Norici), Antintanes, Ardéiens (Ardiei), Pallariens et Japodes, puis Tribales 
(Tribali), Daysiens (Daysii), Istriens (Histri), Libourniens (Liburni), Dalmates (Dalmatae), Curètes (Croates) ", etc. À cette nomenclature s'ajoutent d'autres Slaves, ainsi que les vieilles populations romanes qu'ils avaient repoussées, les Illyriens et les Thraces, ancêtres des Albanais, les Sarmates et les Getes (Getae), peuplades " féroces et hirsutes " d'après la description qu'en fait Ovide lors de son exil dans ces parages, ainsi que les Goths, les Celtes ou même les Francs qui y firent plus d'une incursion. En premier lieu, figurent les anciens Grecs, nos maîtres, sans oublier les Pélasges qui les précédèrent ni même des peuplades moins connues telles des Petchenègues, Guègues, Maniens, Morlaques ou Valaques Noirs (Mauri Volcae), avec tant d'autres que je suis obligé d'omettre dans ce genre d'écrit, faute de place. Dans ce paysage tourmenté, rempli de nos ancêtres et de leurs spectres, qui aurait pu rester "pur " par son origine ou sa «genèse»? Comment y faire, et en fonction de quoi, une «épuration» ou un « nettoyage » ethniques sans recourir à l'absurde ou au crime?

7 Aux différences ethniques et linguistiques s'ajoutent celles de l'imaginaire et des mythologies. Chacun prétend avoir des racines plus profondes que l'autre, des raisons plus convaincantes de s'approprier des territoires plus vastes qu'il ne possède pas encore : de le faire au sein des frontières, jamais tracées avec précision, celles d'un très vieil État plus ou moins hypothétique et d'un ancien pouvoir plongeant ses racines dans les brumes du passé, présenté comme indépendant ou autonome depuis des temps préhistoriques. Les événements réels et leurs représentations fictives se substituent ainsi les uns aux autres. L'histoire et le mythe se confondent - les revendications s'appuient tantôt sur la première, tantôt sur le second, ou bien sur les deux à la fois. Les «arguments» que l'on invoque ou les "preuves » que l'on fournit sont considérés comme irréfutables ou même sacrés. On fait appel, d'un côté, au droit historique, de l'autre, on se réclame du droit naturel. Les uns prétendant détenir la vérité de l'histoire, les autres posséder le droit absolu. Arguments dont les Balkans ont été tant de fois victimes, souvent par leur propre faute.

8 Les historiens traditionnels se sont attachés davantage aux nations qui «arrivent » et « s'installent », qu'à celles qui se fondent sur place ou s'amalgament avec les indigènes et les nouveaux venus. Les querelles ou les affrontements qui en résultent prennent une intensité maximale, et d'ambiguïté aussi, au moment où ces nationalités revendiquent un statut d'État (d'État-nation) - pour rattraper des retards accumulés et se présenter dans leurs anciens habits sur la scène de la modernité.

9 D'autres divergences, tout aussi évidentes, s'entremêlent à ces récits de longue durée. L'une des fractures les plus profondes reste celle que provoqua le schisme chrétien (1054), divisant Églises et croyances, empires et pouvoirs, styles et écritures. Auprès du fossé qui s'est ainsi creusé entre Byzance et latinité, aux confins du christianisme catholique et orthodoxe, s'est inséré l'islam. L'Europe et la Méditerranée se sont scindées et ont éclaté au sein des Balkans. Dans les conflits qui s'y sont déroulés - et qui continuaient de s'envenimer - la foi a été généralement absente, mais non pas la discorde religieuse.

Au cours des siècles, ces différences, adoptées par des fidèles, créaient des divisions qui se transformaient à leur tour en oppositions ou intolérances; ces dernières engendraient l'hostilité ou la haine, devenant par la suite des causes de conflit ou d'incitation à la violence. On peut suivre ainsi, d'une phase à l'autre, l'évolution de ces divergences, tantôt dissimulées par les ombres, tantôt portées au grand jour. Elles comportent des contenus réels, vécus, disséminés dans le temps et l'espace pour être 
finalement détachés de leur source ou de leur nature religieuse. Inscrits dans une conscience rudimentaire ou surtout en l'inconscient collectif, ils se prêtent aux manipulations les plus variées. Les seigneurs de la guerre en ont fait un usage abondant - non seulement au cours des derniers conflits en Bosnie, au Kosovo ou en Croatie, qui ont eu peu en commun avec les guerres de religion au sens propre du terme.

11 La «balkanisation ", elle-même, a partie liée avec des faits et épisodes de cette espèce. La plupart des peuples de la région n'ont pas connu de vraies traditions séculaires. Il ne s'agit pas là uniquement d'un défaut de laïcité face aux croyances: on observe son absence vis-à-vis d'une idée nationale conçue de manière religieuse et, pareillement, à l'égard d'une idéologie (populiste, nationale et surtout nationaliste) transformée en religion. D'une manière analogue, certains secteurs de la culture nationale se transforment en idéologie de la nation. La littérature se réduit à une littérature nationale au sens étroit du terme. Les énergies de l'individu et de la collectivité se voient absorbées par le nationalisme. Ces phénomènes se retrouvent bien au-delà de notre presqu'île infortunée, tout au long des rivages méditerranéens, et ailleurs. Mais cela ne suffit pas pour nous consoler.

12 Après chacun des partages qui se sont opérés dans les Balkans, il restait quelque chose d'inachevé ou de non résolu. Cet inaccomplissement ainsi que l'inadéquation qui en résulte engendrent des situations en porte-à-faux et ambiguës, produisant insatisfaction ou frustration. Une partie de la tâche, parfois la plus importante, reste constamment à faire ou à refaire, à revoir ou à réviser. Reportée à plus tard, à un autre temps " plus favorable». Mais ce temps tarde à venir, ou ne vient jamais. Différentes " vérités " particulières - serbe, bulgare, grecque également, croate, albanaise, turque aussi, catholique, orthodoxe, musulmane, etc. - se sont, chacune, considérées comme seules valables ou équitables. Dans une telle conjoncture, les événements ne peuvent connaitre ni une conclusion ni un aboutissement satisfaisant ou acceptable pour tous. Ils constituent, de manière générale, des périodes instables ou conflictuelles, se rattachant à un passé toujours plus ou moins incomplet ou inabouti. Une histoire tronquée et mutilée, bien plus subie qu'élaborée. Une conscience écornée qui en résulte produit des récits apocryphes, passéistes et souvent partiaux. L'histoire nationale choisit ceux d'entre eux qui lui paraissent les plus utiles et opportuns, évitant l'objectivité ou négligeant les valeurs. Les événements eux-mêmes se voient ainsi déformés, quand ils n'échappent pas à tout contrôle.

13 La conscience nationale, altérée par l'emprise de l'idéologie, construit ses propres scénarios, incitant ses adeptes à les accepter ou à croire en eux. Elle aussi s'appuie davantage sur le mythe que sur la réalité: au point que même les événements fondateurs deviennent souvent prisonniers d'une forme de narration ou de fiction, se refusant à des interprétations rationnelles et critiques.

Les peuples devenus tardivement des nations - et à plus forte raison des États-nations connaissent une certaine dualité : ils se voient et se comportent à la fois en peuple, en nation et éventuellement en État-nation. Il est difficile, dans ce cas, de déterminer des critères d'identification fiables: sommes-nous plus ou moins ceci ou cela, peuple, nation ou État, ressortissants, sujets ou citoyens? Quand et dans quelle mesure sommes-nous l'un ou l'autre? Les terminologies employées à des époques différentes (tribu, communauté, ethnie, peuple et peuplade, nation et nationalité, genèse, attribution ou appartenance, etc.) comportaient en elles-mêmes une part considérable de ces malentendus. 

contredisant, ou encore s'imbriquent l'un sur l'autre de manière factice. Ils constituent souvent des obstacles à de nouveaux processus et aux comportements ultérieurs. Les efforts, somme toute, positifs et légitimes de défendre son propre héritage se trouvent, à un moment donné, face à la nécessité de se défendre soi-même de certains contenus destructifs de ce même héritage. Cela vaut également pour la mémoire: il s'agit, surtout dans les conditions difficiles que traverse une communauté, de la sauvegarder pour survivre avec; or, il arrive la phase ou l'étape où cette communauté doit se sauver elle-même de différentes composantes négatives de sa propre mémoire. Le danger qui apparait en de telles circonstances a été décrit par Jovan Cvijić, l'un des meilleurs spécialistes des Balkans, dans une vaste étude intitulée La péninsule balkanique, écrite au début du vingtième siècle : "Telle l'araignée, les hommes tissent autour d'eux une toile faite de préjugés historiques, de vanités nationales, de modes de vie dénaturés; et cette toile est susceptible de les isoler spirituellement $d u$ reste $d u$ monde et de les rendre archaïques... Les instincts émergeant des périodes précédentes, ceux aussi qui sont les plus primitifs, demeurés jusque-là endormis, commencent à un moment donné à s'éveiller et à agir... » L'avertissement donné par ce scientifique serbe s'est avéré prémonitoire : "l'araignée » a tissé sa toile sur une grande partie de la péninsule balkanique, la couvrant de sang et de tragédie. dont Venise s'était rendue maîtresse, et dans la cité de Dubrovnik, longtemps indépendante, exposée aux fortes influences du littoral Adriatique occidental. Une civilisation brisée par l'invasion ottomane resta pendant plusieurs siècles immergée dans un devenir quasiment informe - un passé privé d'une histoire propre, prenant une part minime ou secondaire dans celle d'autrui. Dans de telles situations, les nations devaient se constituer avec du retard et des interruptions, s'efforçant de récupérer un espace auquel ils croyaient avoir un droit imprescriptible. Les programmes nationaux, même ceux dont les revendications étaient positives en leur propre cadre, restaient le plus souvent perçus par leurs voisins comme autant de menaces ou de conjurations.

Les puissances étrangères tentaient, dès que l'occasion s'en présentait, de stabiliser les situations à l'intérieur des Balkans en déterminant en même temps leurs sphères d'influence. Ainsi, la nouvelle histoire des Balkans a-t-elle été régie par des accords internationaux, qui laissaient derrière eux, en suspens, une multitude de questions irrésolues ou conflictuelles. Un jeu dans lequel la mise ne cesse d'augmenter ou de diminuer, passant d'un partenaire à l'autre. Les exemples en sont nombreux et édifiants. Le traité de Presbourg (l'actuelle Bratislava) livra à Napoléon, en même temps que Venise, le littoral occidental de l'Adriatique et les "provinces illyriennes ». Le Congrès de Vienne laissa l'Autriche occuper tout ce qui, sur ce territoire, avait été sous le sceptre de l'empereur déchu, refusant en même temps d'aller au secours de la Serbie, dont la rébellion fut brisée par le sabre ottoman. Le Congrès de Berlin montra quelque complaisance envers la principauté de Serbie, aux dépens de la Bulgarie, permettant ainsi à la Turquie de se maintenir encore en Bosnie. Les guerres des Balkans se terminèrent par des armistices provisoires, plutôt que par une paix véritable. Le Traité de Versailles favorisa la Serbie, alliée de la France, et permit la création de l'État des Serbes, Croates et Slovènes, qui devint plus tard la Yougoslavie, sans tenir compte des nationalités plus petites qui en faisaient partie (Macédoniens, Monténégrins, Albanais $\mathrm{du}$ Kosovo). Yalta tenta de diviser les zones d'intérêt en deux parties, recherchant une sorte de symétrie, là tout fut désordre et chaos. Sur cette même liste figurent 
également les accords de Dayton, qui mirent fin à la guerre en Bosnie sans assurer pour autant un après-guerre tranquille.

Les espaces balkaniques sont, de toute évidence, jonchés par les vestiges des empires supranationaux et les restes des nouveaux États, découpés au gré des accords internationaux et des programmes nationaux; idées de la nation datant du XIX ${ }^{e}$ siècle et idéologies issues du "socialisme réel » au XX" héritage de deux guerres mondiales et d'une guerre froide, vicissitudes de l'Europe de l'Est et de celle de l'Ouest, relations ambivalentes entre pays développés et ceux " en voie de développement »; tangentes et transversales Est-Ouest et Nord-Sud, liens et ruptures entre la Méditerranée et l'Europe, tentatives d'élargir l'Union européenne par une partie de "l'autre Europe ». Autant de divisions ou de failles, de lignes de partage ou de frontières, matérielles et spirituelles, politiques, sociales, culturelles et autres.

Sur l'échiquier, chaque figure déplacée modifie ainsi l'ensemble des positions et ouvre le jeu vers une autre direction, parfois inattendue. La variété des régimes et des politiques qui en résulte est difficile à classer ou à définir. La Roumanie a subi successivement deux dictatures : fasciste, puis stalino-communiste; elle a une frontière mal tracée avec la Moldavie et, en Transylvanie, une minorité magyare mécontente. Le sort de la Bulgarie, entre une monarchie obsolète et une république de type soviétique, n'est pas sans ressembler, sur plus d'un point, à celui de sa voisine ; elle a dû supporter, en plus, le poids des relations avec sa minorité turque et les tentatives, restées le plus souvent malheureuses, d'un «transfert de population ». L'histoire récente de la Grèce, qui fut à son tour l'objet des accords de Yalta, est marquée par les conséquences de la Résistance et de la collaboration au cours de la Seconde Guerre mondiale ; elle a connu la tentative de renversement communiste et la prise de pouvoir des colonels à tendance fasciste, certains différends au sujet des frontières, accompagnés d'un sentiment d'échec, auquel la mémoire grecque confère un caractère particulièrement dramatique. L'Albanie a subi bien des épreuves, soumise à des pressions sur son littoral et à la lisière du continent, divisée souvent à l'intérieur d'elle-même par ces diverses traditions, restant longtemps séparée de son entourage, assujettie à l'une des dictatures des plus brutales. Quant à la Turquie, sa partie roumélienne a connu, aux marges de son propre État, le sort d'un empire déchu, exposé d'un côté aux ébranlements balkaniques, de l'autre aux convulsions secouant les territoires au-delà du Bosphore, où s'affrontaient une volonté tyrannique et un désir de démocratisation.

Dans la partie balkanique de la Croatie, un nationalisme marqué de traits cléricaux, hostile à la fois à l'orthodoxie et à l'islam, se montre désireux de «fuir les Balkans » à tout prix et de se considérer Mitteleuropéens. Cela ne l'empêche point d'évoquer, non sans quelque orgueil, les principautés ou des petits royaumes croates du Moyen Âge, fondés précisément en deçà des frontières balkaniques et parabalkaniques. Le nationalisme serbe, soutenu par Milošević, a éloigné de la Serbie presque tous ces anciens alliés, non seulement ceux des nationalités slaves, L'agression contre la BosnieHerzégovine, le siège de Sarajevo et le massacre des musulmans près de Srebrenica ont terni l'image de ce pays rebelle et épris de liberté.

On pourrait compléter cette litanie par bien d'autres particularités et de détails tout aussi significatifs : rapports entre la Bulgarie et la Macédoine, tensions entre la Serbie et le Monténégro dans une nouvelle fédération, conflits entre les Kosovars d'origine serbe et albanaise, séparation des nationalités en Bosnie-Herzégovine, désordres intérieurs en Albanie, relations tendues de la Grèce et de la Turquie, question hongroise 
en Transylvanie, roumaine en Moldavie, grecque et turque à Chypre, macédonienne en Grèce, serbe en Croatie, turque en Bulgarie, plus de deux millions d'exilés ou de "déplacés" de l'ex-Yougoslavie, mille et une manières d'assumer et de vivre une «identité postcommuniste», de poser et d'essayer de résoudre la sempiternelle " question nationale » ou bien de réviser des frontières considérées comme «injustes " et «mal tracées ", de s'opposer, en fin de compte, à la fameuse «balkanisation » qui, à l'instar du Destin dans les tragédies nées sous ces ciels, continue de séparer même ce qui paraissait indivis et indivisible. Toute volonté de s'y élargir au désavantage de l'autre se révèle en fin de compte illusoire, ou sombre dans la folie nationaliste : il n'y a pas de place pour une grande Serbie "s'étendant jusqu'à la dernière tombe serbe ", pour une Albanie "s'élargissant vers le nord et l'est », pour une Croatie englobant la Bosnie-Herzégovine "avec une frontière sur la Drina" ou pour une Bulgarie s'annexant la Macédoine ou une partie de la Serbie de l'Est, pour une Grèce empêchant la petite république de Macédoine, déjà assez menacée, de s'appeler simplement la Macédoine. La péninsule s'accommode mal de pareilles ambitions. Elle est trop exiguë pour de telles grandeurs. Ses frontières sont déjà fixées, au-dedans et au-dehors. Les jeux sont faits.

Ce n'est pas seulement dans les Balkans que l'histoire s'écrit en premier lieu comme une histoire nationale. Elle y est communément observée à travers des grilles de lecture trop particulières ou particularistes, souvent folkloriques ou épiques, se fondant sur les sources peu fiables ou des témoignages suspects. Même une défaite ou une blessure peuvent être promues au rang d'« événements fondateurs ", prenant des proportions démesurées au niveau de la conscience nationale ou de l'imaginaire collectif. Pour n'en donner qu'un exemple, lié à l'actualité la plus brûlante, il suffit de rappeler le cas du Kosovo. Les questions concernant son passé, son appartenance ou son statut présent sont posées bien différemment par les historiens ou les politiciens appartenant aux nations qui y cohabitent, et par ceux dont l'origine n'est ni serbe ni albanaise. Leurs arguments, même lorsqu'ils partent des mêmes faits, aboutissent généralement à des conclusions divergentes. Cet exemple et la leçon qu'on peut en tirer méritent une place dans ce récit.

Le passé préhistorique des Balkans ne pose heureusement pas de problème : le Kosovo fut autrefois un grand lac dont la nature garde des traces; le fleuve Ibar a emporté ses eaux vers la mer Noire, la rivière de Lepenac vers l'Égée, laissant tout au long de leurs lits des massifs abrupts et, au centre, des vallées verdoyantes. Le nom de Kosovo polje signifiant «champ des merles" (campus turdorum), apparaît dès le Moyen Âge. Les ancêtres des Albanais, Illyriens ou Thraces y ont habité dès la fin du $\mathrm{III}^{\mathrm{e}}$ millénaire avant l'ère chrétienne. Ptolémée signale, au $\mathrm{II}^{\mathrm{e}}$ siècle de notre ère, la présence des Albanoi entre les montagnes de l'ancienne Dardanie et de la Macédoine. Les Slaves (Serbes en particulier, Bulgares et Macédoniens aussi) sont arrivés aux $\mathrm{VI}^{\mathrm{e}}-\mathrm{VII}^{\mathrm{e}}$ siècles après J.-C. dans cette région, alors parcourue, entre autres, par des Valaques (en partie descendants des colons romains) et d'autres peuplades nomadisant aux alentours. Cet espace est devenu, du XII ${ }^{e}$ au XIV ${ }^{e}$ siècle, « le cœur » du royaume médiéval serbe : l'État de Rascie (Raška - ancien nom de la Serbie), après avoir conquis certaines terres byzantines, s'y établit en 1180 ; le tsar Dušan, nommé «Le Puissant » (Silni), fonde sa résidence à Prizren, au centre de la région ; l'archevêque puis le patriarche s'installent à Peć et y construisent le fameux monastère de Gračanica. Le roi Stephan Uroš II (1282-1321) s'intitule « roi de la Serbie, de Dioclea (actuel Monténégro), d'Albanie et de la côte ». Telle est la situation à la veille de la bataille de Kosovo (1389), dans laquelle les 
Serbes subirent une grandiose défaite face à la puissante armée ottomane. Leurs voisins balkaniques, parmi lesquels se trouvait aussi un certain nombre d'Albanais, leur furent d'un modeste secours. (Je cite par endroits les recherches de l'historien français Georges Castellan, spécialiste des Balkans, dont les jugements paraissent plus crédibles $\mathrm{du}$ fait qu'il n'appartient à aucune des nations de la péninsule.) «N'ayant pas devant les yeux le souvenir d'un passé glorieux ", les Albanais adhérèrent plus facilement à la foi des vainqueurs et "fournirent au Sultan un nombre imposant de serviteurs dévoués ». Quant aux Serbes, ils furent contraints d'effectuer une «Grande migration » (Velika seoba) sans pour autant abandonner complètement la région.

En 1690, l'armée autrichienne pénétra dans ce territoire kosovar jusqu'à Peć, invitant par une proclamation les "Serbes, Albanais, Mésiens, Bulgares, Illyriens, Macédoniens et Rasciens " à se soulever contre les Ottomans. Les Serbes y jouèrent un rôle important, guidés par le patriarche Arsenié III Crnojević. Les insurgés durent bientôt se replier et émigrer à nouveau (certaines sources parlent d'environ 70.000 à 80.000 personnes), bénéficiant de l'asile qui leur fut accordé par Léopold ${ }^{\mathrm{er}}$ dans ses terres. Aussi leur nombre au Kosovo fût-il, une fois de plus, fortement réduit.

En 1903, le consulat austro-hongrois de Prizren recensa, on ignore par quel procédé, la population et y dénombra $45 \%$ de Serbes contre $55 \%$ d'Albanais. Il s'agit très probablement d'un chiffre approximatif. La zone allait faire l'objet non seulement des visées de la Serbie, qui invoquait un droit historique à son appartenance, mais aussi des projets expansionnistes autrichien et italien. Au cours des guerres des Balkans (1912-1913), elle fut occupée par l'armée serbe. À la suite de la «Grande Guerre » de 1914-1918, elle devint partie intégrante du Royaume de Yougoslavie. L'entre-deuxguerres y vit l'exode des habitants turcs, suivi d'une « réforme agraire » qui privilégiait les Serbes et les Monténégrins au détriment des «shipetars» (Albanais), couche sociale la plus démunie et culturellement la plus arriérée. Au cours de la Deuxième Guerre mondiale, l'Italie fasciste s'empara de ce territoire et le réunit à l'Albanie : un simulacre de la «Grande Albanie » apparut à l'horizon d'une population désunie depuis les temps immémoriaux, dirigée par une oligarchie corrompue, à la solde de l'étranger. Après la capitulation italienne (1943), bien des Albanais, désorientés politiquement, continuèrent de "collaborer " avec les nouveaux occupants et s'enrôlèrent dans des troupes nommées "balistes", dirigées par les nazis. Le châtiment qu'ils subirent au début de l'après-guerre se distingua par une particulière cruauté à leur égard et laissa des traces profondes dans leur mémoire.

Une fédération balkanique, souhaitée par Staline, où tous les Albanais seraient soumis à leurs voisins plus forts et influents fut adroitement évitée par Tito et Dimitrov. Après la rupture de la Yougoslavie avec l'U.R.S.S. (1948) et l'ouverture de ses frontières, un nombre considérable d'ouvriers kosovars partirent vers l'Occident. Menant une vie à la fois laborieuse et parcimonieuse, ils réussirent à acquérir des moyens pour racheter, selon les traditions ancestrales, des parcelles considérables de la terre au Kosovo et atteindre un taux de croissance le plus élevé en Europe. Les Serbes de la région se virent alors de plus en plus poussés vers un lent et inexorable exode : il en restait environ $10 \%$ contre $90 \%$ d'Albanais, avant le début de la toute dernière guerre et la "purification ethnique» qui y fut pratiquée. Nous ne disposons pas de données tout à fait précises concernant la période actuelle, au début d'un nouveau millénaire. Mes tentatives d'entreprendre des recherches dans la région se sont avérées vaines et irréalisables. 
Les événements récents reflètent, entre autres, un imaginaire politique quasiment paradoxal : au moment où Tito fut accusé par les ultranationalistes Croates d'avoir " trahi sa nation (croate)» et que le nationalisme grand-serbe l'incriminait d'avoir détruit (en tant que Croate) "l'intégrité de la Serbie», seuls les Kosovars d'origine albanaise, accusés de séparatisme, portaient à la tête de leurs défilés protestataires l'effigie du maréchal unificateur. Dans une « région autonome » de l'ex-Yougoslavie, ils avaient connu une vie incomparablement plus libre et prospère que leurs frères d'une Albanie soumise à la tyrannie ubuesque d'Enver Hoxha. Après avoir été des victimes de la répression des milices de Milošević, les Kosovars albanais, devenus une majorité écrasante de la région, exercent sur la minorité serbe resserrée autour de quelques temples et monastères, une pression insupportable. Maints ouvrages parus à Belgrade ne cessent d'évoquer certains us et coutumes connus dans les traditions tribales des Albanais: "la levée de sang» (gjakmarrja) qu'ils pratiquaient dans le passé ou les vengeances atroces, requises par leurs « Canons » ancestraux (Kanuni i Lekë Dukagjinit).

Je reprends ici la métaphore initiale des mouvements telluriques, un témoignage fourni par un moine byzantin, Georges Pachymère de son nom, décrivant un séisme subi par la ville albanaise de Dyrrachion (Durrës): "C'était une secousse sous forme de pulsations successives... Des chocs inhabituels remuaient la terre sans discontinuer, bruits que, en langage commun, on appellerait gémissements et qui étaient le signe évident d'un malheur tout proche ». De telles descriptions complètent probablement les épisodes historiques que nous venons d'évoquer.

De telles situations abondent des sujets particulièrement pénibles, difficiles à aborder : celui de la cruauté, dont les images prises sur le vif ont récemment parcouru le monde. Certains refusent d'en parler pour ne pas offenser une population dont la majorité n'est nullement responsable; d'autres, originaires de ces régions, préfèrent se taire - ayant honte. Rappelons malgré tout l'une des scènes les plus atroces de la littérature de notre siècle. L'un des premiers chapitres du Pont sur la Drina, œuvre maitresse d'Ivo Andrić (écrivain d'origine croate et bosniaque, Serbe d'adoption et Yougoslave par vocation, Prix Nobel de littérature) décrit le supplice du pal infligé à un Slave rebelle sous l'occupation ottomane : " un pal de chêne, de près de quatre archines de long, taillé en pointe comme il convient, le bout ferré parfaitement effilé, enduit de suif»; un homme "empalé comme un agneau sur la broche, sauf que la pointe ne ressortait pas par la bouche, mais dans le dos, sans avoir gravement endommagé ni les intestins, ni le cœur, ni les poumons ». L'opération requiert la compétence des "spécialistes » et l'usage des instruments appropriés plusieurs marteaux ou maillets qui poussent progressivement, coup par coup, le pieu dans le corps dénudé, d'un bout à l'autre, de l'entrejambe vers les omoplates. Il s'agit d'éviter les lésions des organes vitaux afin que le supplicié, pour inspirer l'effroi et donner l'exemple aux autres, survive ainsi quelques jours : " gonflé et rouge, ressemblant à un masque, râlant du haut du pal et crachant une écume blanche ».

Il faut imaginer bien des cas semblables le long des routes boueuses des Balkans, au milieu des carrefours tortueux et bariolés, au cours des siècles qui se succédaient sans se conclure. La souffrance et « le mal intériorisé » de la sorte, la révolte ou la vengeance qu'ils suscitent ou réclament, tout ceci n'est pas " gardé » ou « conservé » seulement à l'intérieur du corps ou au fond de la mémoire, mais aussi quelque part ailleurs - nous ne savons exactement ni où ni comment. Les circonstances réveillent un jour ces états troubles et traumatisants, les font revivre sous une forme de résistance ou d'agression, de tourment ou de cruauté. 

Vienne n'a jamais été conquise "par les hordes asiatiques", tout comme Venise ou Trieste ou n'importe quelle autre cité italienne : sans ces sacrifices, il n'y aurait pas eu de Renaissance en Italie ni de prospérité de la Mitteleuropa. "Nous l'avons payée leur liberté par notre sang ". Nous avons contribué ainsi à "sauver l'Europe et sa civilisation ». Plus au nord, ce furent « nos frères russes » qui opposaient un bouclier analogue, plus résistant encore, aux cruelles invasions venues des steppes d'outre-Oural, protégeant ainsi les pays qui allaient devenir la partie la plus avancée du continent. acceptais quelques-uns de ses arguments avec un certain orgueil. Je ferme ce chapitre, conscient des conclusions auxquelles peuvent conduire de semblables thèses. Certaines expériences tragiques, comme celles qui viennent d'être relatées, persistent longtemps au sein d'une tribu ou d'une nation. Leur survie dépend de circonstances qui ne sont pas seulement historiques. Si je les mentionne, ce n'est pas pour condamner ni amnistier qui que ce soit. C'est juste pour en tenir compte.

En dehors et au-delà de cet inventaire, il faudrait faire état d'une riche production littéraire et artistique, véritables trésors qui se sont constitués en dépit des conditions dont il a été question. J'ai déjà mentionné les noms d'Andrić et Krleža (ce dernier, né à Zagreb, n'a jamais perdu de vue la réalité balkanique). Le romancier serbe Miloš Crnjanski mérite sa place à leurs côtés, de même que mon défunt ami Danilo Kiš, "hybride juivo-monténégrin", comme il lui plaisait de s'appeler, Yougoslave et Européen à part entière. Les Grecs Nikos Kazantzakis, par sa prose, Séféris ou Ritsos par leur poésie se montrent dignes du grand héritage hellénique. L'Albanie nous a donné un romancier de génie, Ismail Kadare, qui compte parmi les plus importants dans les lettres contemporaines de l'Europe. Ivan Vazov et Georgi Karaslavov ont tracé une voie royale au roman bulgare que d'autres, prosateurs et poètes, ont pu suivre après eux. Les poètes macédoniens Aco Šopov et Blaže Koneski ont contribué par leurs œuvres à codifier la langue de leur nation. Nazim Hikmet, Jachar Kemal ou le jeune Orham Pamouk sont également lus et appréciés des deux côtés du Bosphore pour leurs œuvres ou leurs exemples. La littérature roumaine a dépassé ses propres frontières et a affirmé, entre autres, quelques grands auteurs de langue française: Panaït Istrati, "métèque rouméno-grec ", Tzara, Ionesco, Cioran... J'interromps cette énumération qui, dans les limites de ce récit, ne peut éviter de rester partielle, voire partiale.

C'était là l'une des nombreuses manières de voir et de présenter les Balkans, « cet espace qui produit plus d'histoire qu'il n'en peut consommer » (W. Churchill) et qui transforme ces excédents en excès pour mieux se détruire. Selon les uns, c'est « la vitrine " de notre continent, son «thermomètre " selon les autres. D’un côté «le berceau de l'Europe », de l'autre sa « poudrière ». 


\section{RÉSUMÉS}

Les Balkans témoignent de contradictions géographiques et politiques, perceptibles dans le changement d'appellation des lieux au cours de l'Histoire : de la péninsule illyrienne, grecque, byzantine à la « Turquie d'Europe » plus récente. Il est resté une dénomination venue des Turcs, les «Balkans ». Cette presqu'île n'offre pas de frontières naturelles difficiles à franchir. Les délimitations des géographes et historiens sont relatives et souvent arbitraires, et donc contestées. Sa définition est difficile, car, étant associée à la progression des troupes ottomanes, cette «zone de turbulences " garde aux yeux de certains un caractère négatif. Marqués par les bouleversements telluriques et historiques, les Balkans témoignent d'une pluralité et d'une variété démographiques, ce qui pose un grand nombre de questions en termes de rapports ethniques, de constructions identitaires et territoriales. Les revendications s'appuient aussi bien sur le mythe que sur l'Histoire. L'apparition de l'État-nation a constitué dans ces régions de multiples fractures à l'image du schisme chrétien de 1054, divisant Églises et croyances, empires et pouvoirs, styles et écritures. L'islam a engendré de nouvelles oppositions ou intolérances. Les puissances étrangères sont intervenues dans ce jeu pour tenter de le stabiliser tout en déterminant leurs sphères d'influence, figeant par le jeu des traités une multitude de questions irrésolues ou conflictuelles, sans apporter de réponse durable. Cet inachevé, dans une histoire faite de partages, suscite des frustrations, et nécessite une réécriture qui tarde à se faire jour, faute d'apaisement entre les différentes sensibilités. La littérature se fait l'écho de ces situations par l'évocation de la cruauté, sous l'occupation ottomane (cf. Ivo Andrić) notamment, mais aussi dans l'actualité récente. "Cet espace qui produit plus d'histoire qu'il n'en peut consommer » (W. Churchill) est le cadre de création d'une riche production littéraire et artistique.

The Balkans are full of geographical and political contradictions, which becomes obvious by simply noticing the fact that their name has changed more than once during the course of history: starting as Illyrian peninsula, it was then transformed into Greek, then Byzantine peninsula while recently it is known as "European Turkey". Originally, the "Balkans » is a name that came from the Turks. This almost island has natural frontiers which are easy to cross. The boundaries proposed by geographers and historians are relative and often arbitrary, thus contested. Defining the Balkan frontiers may prove to be a task that is hard to accomplish, since being associated with the progression of the ottoman troops, this "zone of turbulence" holds, for some, a negative connotation by itself. Marked by geographical and historical upheaval, the Balkans constitute an area of demographical plurality and variety which in hand creates a number of questions and problems with regard to ethnical relations as well as the creation of identity, territorial or other. All claims made were relying in mythology as well as in historical facts. As the Nation State made its appearance as a formation in the region it created even more fragmentation, almost imitating the one caused by the great schism of 1054, where geographical space was used as a way to separate Churches, beliefs, empires, powers as well as styles of art or writing. The appearance of Islam, in the area, has generated new oppositions and intolerances. Foreign forces have intervened in this already fragile balance in an effort to provide stability, while determining their own spheres of influence, thus freezing with their interference (manifested through the signing of numerous treaties), a number of issues that were left unresolved, or controversial, without successfully bringing any durable solutions to the matter. These series of unresolved issues, in an area which has a history of division, promote frustration and need a revision, even a belated one, in order to satisfy and appease the different sensitivities that present themselves with regard to the Balkans. Literature becomes the echo of such questions, as it evokes the cruelty suffered under the ottoman occupation (see Ivo Andrić), as 
well as in more recent events. The Balkans, which "produce more history than they can consume", as W. Churchill has once put it, become the canvas for a rich literary and artistic production.

INDEX

Mots-clés : caractères balkaniques

Keywords : Balkanic characteristics, Balkans 